\title{
Double-copy constructions and unitarity cuts
}

\author{
Zvi Bern, ${ }^{1,2}$ Scott Davies, ${ }^{2}$ and Josh Nohle ${ }^{3}$ \\ ${ }^{1}$ Department of Physics, CERN Theory Division, CH-1211 Geneva 23, Switzerland \\ ${ }^{2}$ Department of Physics and Astronomy, University of California at Los Angeles, \\ Los Angeles, California 90095, USA \\ ${ }^{3}$ Nordita, KTH Royal Institute of Technology and Stockholm University, \\ Roslagstullsbacken 23, SE-10691 Stockholm, Sweden \\ (Received 1 November 2015; published 9 May 2016)
}

\begin{abstract}
The duality between color and kinematics enables the construction of multiloop gravity integrands directly from corresponding gauge-theory integrands. This has led to new nontrivial insights into the structure of gravity theories, including the discovery of enhanced ultraviolet cancellations. To continue to gain deeper understandings and probe these new properties, it is crucial to further improve techniques for constructing multiloop gravity integrands. In this paper, we show by example how one can alleviate difficulties encountered at the multiloop level by relaxing the color-kinematics duality conditions to hold manifestly only on unitarity cuts instead of globally on loop integrands. As an example, we use a minimal Ansatz to construct an integrand for the two-loop four-point nonsupersymmetric pure Yang-Mills amplitude in $D$ dimensions that is compatible with these relaxed color-kinematics duality constraints. We then immediately obtain a corresponding gravity integrand through the double-copy procedure. Comments on ultraviolet divergences are also included.
\end{abstract}

\section{INTRODUCTION}

The duality between color and kinematics [1,2] offers a practical means for obtaining difficult-to-construct higher-loop scattering amplitudes in gravity theories. This duality, conjectured by Carrasco, Johansson and one of the authors (BCJ) to hold to all loop orders, stipulates that there exist forms of amplitude integrands where the kinematic numerators of all diagrams satisfy the same algebraic relations as the color factors. BCJ duality was first formulated for adjoint-representation states and has recently been generalized to also include fundamental-representation states [3,4]. Once the duality is manifest in a gauge-theory amplitude, corresponding gravity amplitude integrands are obtained simply by replacing gauge-theory color factors with dualitysatisfying kinematic numerators. This is known as the "double-copy" construction of gravity. It effectively reduces the problem of obtaining multiloop gravity integrands to the much simpler problem of finding color-kinematics duality-satisfying gauge-theory integrands. The duality also imposes a rigid structure on gauge-theory loop integrands that can greatly streamline their construction [5], including nonplanar contributions.

At loop level, BCJ color-kinematics duality remains a conjecture. Moreover, as yet there is no constructive means

Published by the American Physical Society under the terms of the Creative Commons Attribution 3.0 License. Further distribution of this work must maintain attribution to the author(s) and the published article's title, journal citation, and DOI. for finding forms of the integrands where the duality is manifest. Instead, higher-loop integrands are generally constructed case by case using Ansätze [2,5,6], whose generalized unitarity cuts are then matched to those of the desired amplitude via the unitarity method $[7,8]$. There has, however, been important progress in a variety of directions, including understanding the underlying group-theoretic structure behind the duality [9], explicit constructions of loop integrands $[2,5,6,10-12]$, identifying the duality in classical solutions including those for black holes [13], expanding the range of theories where the duality applies $[3,4,14]$, as well as various other studies [15]. Recent work based on twistor string theory and scattering equations also offers a new avenue for constructing gravity loop amplitudes that manifest the double-copy structure [16].

In this paper, we explore a different strategy for improving our ability to construct multiloop gravity amplitudes. A generic problem with using Ansätze to construct kinematic numerators is they may not be general enough. This issue is important for state-of-the-art calculations: For example, it has proven difficult to construct integrands for the five-loop four-point amplitude of $\mathcal{N}=4$ super-YangMills theory that manifest BCJ duality between color and kinematics [17]. Here we will give a two-loop example in pure Yang-Mills theory that runs into similar difficulties, where a seemingly reasonable Ansatz is not compatible with both global BCJ duality and unitarity constraints. By "global" BCJ constraints, we mean the full set of BCJ duality constraints on the integrand. Instead of expanding the Ansätze - for instance by allowing for nonlocalities or abandoning relabelling symmetries-the solution we adopt 
here is to relax the BCJ duality constraints so that they hold only on a spanning set of unitarity cuts. A "spanning set" of unitarity cuts refers to a set of unitarity cuts sufficient for constructing all terms in the amplitude. We can then employ the simpler Ansatz while maintaining the key double-copy property, thus allowing us to obtain corresponding gravity loop integrands directly from gaugetheory ones.

To demonstrate the usefulness of this approach, we utilize it to construct the $D$-dimensional two-loop fourgluon integrands of pure Yang-Mills theory in a form compatible with the double-copy construction of gravity. The corresponding gravity amplitude is for a theory of gravity coupled to a dilaton and an antisymmetric tensor. As a warm-up, we first look at the case of fourdimensional identical-helicity external gluons. A loop integrand satisfying manifest global BCJ duality was already given in Ref. [18]. We instead choose to work with an earlier form of the integrand [19] that displays exactly the property that the duality is not manifest on the integrand but is instead manifest on a spanning set of generalized unitarity cuts. For general external-leg polarizations in $D$ dimensions, there is currently no known representation of the amplitude where global BCJ duality is manifest. We first show that a natural "minimal Ansatz" is not compatible with both unitarity and manifest global BCJ constraints on the integrand. Enlarging an Ansatz can quickly become a losing game because of the rapid proliferation of possible terms. The minimal Ansatz we use has locality, manifest crossing symmetry and loopby-loop power counting no worse than that of ordinary Feynman diagrams. By loop-by-loop power counting, we are referring to the maximum number of powers of each independent loop momentum that can appear in the numerator of a given diagram. We show that once we relax BCJ duality constraints so that they are manifest only on generalized unitarity cuts instead of the full integrand, the duality and unitarity constraints are all compatible. Having the duality manifest in the cuts ensures that the gravity integrand constructed through the double-copy procedure will have the correct gravity cuts. The Yang-Mills integrand we obtain therefore has the double-copy property and achieves the goal of immediately giving us a corresponding gravity integrand with a variety of desirable properties inherited from the YangMills integrand.

Finding improved means for constructing multiloop gravity integrands is important for studies of ultraviolet properties of gravity theories. (For a recent update see Ref. [17].) Explicit calculations show that gravity theories have a softer ultraviolet behavior than known standardsymmetry considerations predict. Certain gravity amplitudes possess "enhanced cancellations" [20], which are defined to be ultraviolet cancellations that cannot be exhibited diagram by diagram in any covariant formalism.
(By a covariant formalism, we mean that the only kinematic denominators in each diagram are those of standard Feynman propagators.) This is a new phenomenon not accounted for by standard-symmetry considerations. In particular, $\mathcal{N}=4$ supergravity [21] at three loops [22] and $\mathcal{N}=5$ supergravity at four loops [20] are ultraviolet finite. No satisfactory standard-symmetry explanation has been found as yet for these cases, despite some effort [23]. By four loops, $\mathcal{N}=4$ supergravity does have an ultraviolet divergence [24], but it has a curious structure connected to a duality-symmetry anomaly [25] not present in $\mathcal{N} \geq 5$ supergravities. In fact, as recently shown, divergences in gravity are much more subtle than symmetry considerations suggest: They can be modified at leading order by evanescent effects and change under duality transformations [26]. ("Evanescent effects" refer to effects that vanish strictly in $D=4$ but lead to nontrivial contributions in dimensional regularization.) These results emphasize the need for improved, more powerful methods to study the various surprising phenomena that become visible only at the multiloop level. The present paper is a modest step in this direction.

This paper is organized as follows. In Sec. II, we first review the duality between color and kinematics before describing our approach of applying it to generalized unitarity cuts. Then in Sec. III, we present the identicalhelicity two-loop four-point amplitude as a warm-up to the case with general polarizations, which is subsequently discussed in Sec. IV. In Sec. V, we give the two-loop ultraviolet divergence of the gravity theory, extracted from the identical-helicity amplitude. We present our conclusions in Sec. VI.

\section{DUALITY BETWEEN COLOR AND KINEMATICS}

In this section, we first review BCJ duality between color and kinematics [1,2] and then explain our procedure for imposing it on unitarity cuts. At loop level, the duality remains a conjecture, but even so it can greatly streamline the construction of loop amplitudes [5] for two reasons:

(1) It imposes a structure on gauge-theory amplitudes that can be exploited to determine the full loop integrand from a small subset of diagrams called "master diagrams."

(2) Once a form of gauge-theory loop integrands has been found where the duality is manifest, corresponding gravity integrands are easily constructed by replacing color factors with gauge-theory numerators that satisfy the duality.

The unitarity method [7] then offers a convenient way to confirm that the construction is correct. This needs to be done case by case since $\mathrm{BCJ}$ duality remains a conjecture at loop level. 
Here we propose applying a less powerful form of the duality that retains the above second property at the expense of losing the first one. We impose that the duality is manifest on generalized unitarity cuts that decompose loops into trees and relax the condition that it be manifest in full loop integrands. This strategy can be helpful for constructing gravity integrands whenever it is difficult to find expressions that are compatible with both unitarity and manifest global BCJ duality in the full integrand.

\section{A. Tree level}

An $m$-point gauge-theory tree amplitude with all particles in the adjoint representation can always be written as

$$
\mathcal{A}_{m}^{\text {tree }}=g^{m-2} \sum_{j} \frac{c_{j} n_{j}}{\prod_{\alpha_{j}} p_{\alpha_{j}}^{2}},
$$

where the sum over $j$ is over the set of distinct $m$-point graphs with only cubic vertices. Inequivalent relabelings of a given diagram are counted as distinct graphs, and $g$ is the gauge coupling constant. Associated with each graph $j$ are the following:

(i) $1 / \prod_{\alpha_{i}} p_{\alpha_{j}}^{2}$ : The Feynman propagators affiliated with the graph. (The associated factors of $i$ are absorbed into the numerators.)

(ii) $c_{j}$ : The color factor obtained by dressing every vertex of the graph with the group-theory structure constant, $\tilde{f}^{a b c}=i \sqrt{2} f^{a b c}=\operatorname{Tr}\left(\left[T^{a}, T^{b}\right] T^{c}\right)$, where the Hermitian generators of the gauge group are normalized via $\operatorname{Tr}\left(T^{a} T^{b}\right)=\delta^{a b}$.

(iii) $n_{j}$ : The numerator that contains the nontrivial kinematic information, including momenta, polarizations and possibly spinors.

To obtain the form in Eq. (2.1), we convert contributions from contact-term diagrams - those with higher-than-threepoint vertices - to ones with only cubic vertices by multiplying and dividing by the appropriate propagators, i.e., inserting factors of $p_{\alpha}^{2} / p_{\alpha}^{2}=1$.

A nontrivial task is to find kinematic numerators that satisfy the duality between color and kinematics. The numerators appearing in Eq. (2.1) are by no means unique due to the freedom to move terms between different diagrams, also known as generalized gauge invariance $[1,2,27,28]$. This freedom can be utilized to find representations of the amplitude where the kinematic numerators obey the same algebraic relations that the color factors obey $[1,2]$. For adjoint representations in ordinary gauge theories, this is simply the Jacobi identity,

$$
c_{i}=c_{j}-c_{k} \Rightarrow n_{i}=n_{j}-n_{k},
$$

where $i, j$ and $k$ label three diagrams whose color factors obey the Jacobi identity. The basic Jacobi relation is displayed in Fig. 1. The generalization of the identity to $m$-point tree-level amplitudes is seen diagrammatically by

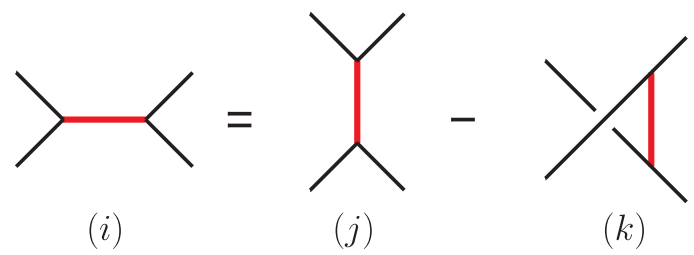

FIG. 1. The basic Jacobi relation for either color or numerator factors. These three diagrams (i), (j) and (k), corresponding to color factors $c_{i}, c_{j}$ and $c_{k}$ respectively or numerator factors $n_{i}, n_{j}$ and $n_{k}$ respectively, can be embedded in a larger diagram at tree level or loop level. The propagator around which the Jacobi relation is performed is shaded (red).

embedding Fig. 1 in larger diagrams, where the other parts of the three diagrams remain unaltered under the duality. Furthermore, whenever the color factor of a diagram is antisymmetric under a swap of legs, we require that the numerator obey the same antisymmetry,

$$
c_{i} \rightarrow-c_{i} \Rightarrow n_{i} \rightarrow-n_{i} .
$$

At tree level, numerators that obey the duality for any number of external legs are known [29]. The numerator relations are nontrivial functional relations because they depend on momenta, polarizations and spinors, as discussed in some detail in Refs. [5,30].

A central aspect of the duality is the ease with which gravity amplitude integrands follow from gauge-theory ones once the duality is made manifest $[1,2]$ : One simply replaces the color factor of a gauge-theory amplitude with a kinematic numerator, $\tilde{n}$, from a second gauge theory which has the duality manifest:

$$
c_{i} \rightarrow \tilde{n}_{i}
$$

With this replacement in Eq. (2.1), we obtain the doublecopy form of gravity tree amplitudes,

$$
\mathcal{M}_{m}^{\text {tree }}=i\left(\frac{\kappa}{2}\right)^{m-2} \sum_{j} \frac{\tilde{n}_{j} n_{j}}{\prod_{\alpha_{j}} p_{\alpha_{j}}^{2}},
$$

where $\tilde{n}_{j}$ and $n_{j}$ are gauge-theory numerator factors and the gravitational coupling is given in terms of Newton's constant via $\kappa^{2}=32 \pi G_{N}$. A tree-level proof for this construction is given in Ref. [28]. We note that in the double-copy procedure, only one of the two sets of numerators needs to satisfy the duality of Eq. (2.2) $[2,28]$. The specific gravity theory one obtains depends on which gauge theories are used as inputs into the construction.

\section{B. Loop level}

At loop level, the duality (2.2) remains a conjecture [2]. As at tree level, we express the amplitudes as a sum over diagrams with only cubic vertices: 


$$
\mathcal{A}_{m}^{L \text {-loop }}=i^{L} g^{m-2+2 L} \sum_{\mathcal{S}_{m}} \sum_{j} \int \prod_{l=1}^{L} \frac{d^{D} p_{l}}{(2 \pi)^{D}} \frac{1}{S_{j}} \frac{c_{j} n_{j}}{\prod_{\alpha_{j}} p_{\alpha_{j}}^{2}} .
$$

The first sum runs over the $m$ ! permutations of the external legs, denoted by $\mathcal{S}_{m}$. The $S_{j}$ symmetry factor removes any overcounting from these permutations and also from any internal automorphism symmetries of graph $j$. Here the $j$-sum runs over the set of distinct, nonisomorphic, $L$-loop $m$-point graphs with only cubic or trivalent vertices. Again, absorbing numerators of contact diagrams that contain higher-than-three-point vertices into numerators of diagrams with only trivalent vertices is trivial. However, it is nontrivial to make a rearrangement into the $\mathrm{BCJ}$-conjectured form, where the numerator factors obey the same Jacobi relations (2.2) and symmetry properties (2.3) as the color factors.

The generalization of BCJ duality to loop-level amplitudes is to embed Fig. 1 in larger loop diagrams [2]. We then demand that the numerators of all diagrams obey the BCJ relations (2.2) and (2.3). We refer to this as "global BCJ duality." Using the kinematic Jacobi relations, one can solve for the numerators of all diagrams in terms of a relatively small number of "master" numerators [5].

Once we have gauge-theory numerator factors that satisfy the duality, the substitution of color factors by the numerator factors (2.4) gives us the double-copy form of gravity loop integrands,

$\mathcal{M}_{m}^{L \text {-loop }}=i^{L+1}\left(\frac{\kappa}{2}\right)^{m-2+2 L} \sum_{\mathcal{S}_{m}} \sum_{j} \int \prod_{l=1}^{L} \frac{d^{D} p_{l}}{(2 \pi)^{D}} \frac{1}{S_{j}} \frac{\tilde{n}_{j} n_{j}}{\prod_{\alpha_{j}} p_{\alpha_{j}}^{2}}$,

where $\tilde{n}_{j}$ and $n_{j}$ are gauge-theory numerator factors. As at tree level, the theories to which the gravity amplitudes belong are dictated by the input gauge theories. The gravity theory corresponding to two copies - call them "left," $L$, and "right," $R$, copies — of nonsupersymmetric pure Yang-Mills theory includes a graviton, a dilaton and an antisymmetric tensor field. We can see this by decomposing the product of two gluon polarization vectors into irreducible parts: a symmetric and traceless term, an antisymmetric term and a trace term. For instance, for a $D$-dimensional external leg with momentum $k$ and polarization vectors $\varepsilon_{L}, \varepsilon_{R}$ with reference momentum $q$, we can write

$$
\begin{aligned}
\varepsilon_{L}^{\mu} \varepsilon_{R}^{\nu}= & {\left[\frac{1}{2}\left(\varepsilon_{L}^{\mu} \varepsilon_{R}^{\nu}+\varepsilon_{L}^{\nu} \varepsilon_{R}^{\mu}\right)\right.} \\
& \left.-\frac{1}{D-2}\left(\eta^{\mu \nu}-\frac{k^{\mu} q^{\nu}+k^{\nu} q^{\mu}}{k \cdot q}\right) \varepsilon_{L} \cdot \varepsilon_{R}\right] \\
& +\frac{1}{2}\left(\varepsilon_{L}^{\mu} \varepsilon_{R}^{\nu}-\varepsilon_{L}^{\nu} \varepsilon_{R}^{\mu}\right) \\
& +\frac{1}{D-2}\left(\eta^{\mu \nu}-\frac{k^{\mu} q^{\nu}+k^{\nu} q^{\mu}}{k \cdot q}\right) \varepsilon_{L} \cdot \varepsilon_{R} .
\end{aligned}
$$
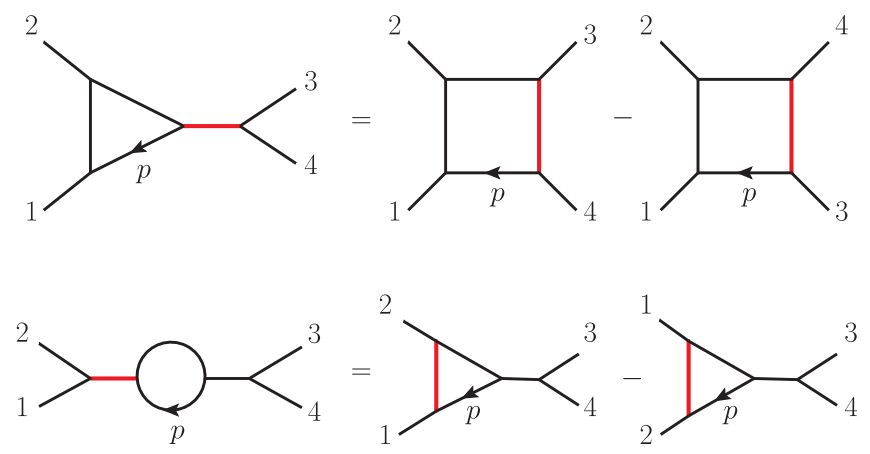

FIG. 2. The Jacobi relations determining the triangle and bubble numerators in terms of box numerators. The shaded (red) propagator indicates the line around which the Jacobi identities are applied.

These three terms correspond to the polarization tensors of a graviton, an antisymmetric tensor field and a dilaton, respectively. Note that $\eta^{\mu \nu}-\left(k^{\mu} q^{\nu}+k^{\nu} q^{\mu}\right) /(k \cdot q)$ is the usual projector for the $(D-2)$-dimensional space spanned by the polarization vectors orthogonal to both $k$ and $q$.

As a simple illustration of color-kinematics duality at loop level, consider the BCJ numerator identities for oneloop four-point amplitudes:

$$
\begin{gathered}
n_{12(34) ; p}^{(1)}=n_{1234 ; p}^{(1)}-n_{1243 ; p}^{(1)}, \\
n_{(12)(34) ; p}^{(1)}=n_{12(34) ; p}^{(1)}-n_{21(34) ; p}^{(1)},
\end{gathered}
$$

corresponding to each row of Fig. 2. By $n_{1234 ; p}^{(1)}$ we mean the box numerator with the external legs following the cyclic ordering 1234 and $p$ is the loop momentum. Similarly, $n_{(12) 34 ; p}^{(1)}$ and $n_{(12)(34) ; p}^{(1)}$ denote triangle and bubble numerators corresponding to the leftmost diagrams in Fig. 2. (Here we do not consider the bubble-on-externalleg or tadpole diagrams that vanish in dimensional regularization after integration.) Since the numerators of the other diagrams can be derived from the box diagrams, we call the box diagrams master diagrams. If we impose that the numerators obey manifest crossing symmetry, i.e., that the different box numerators are obtained from each other simply by appropriate relabelings of the legs, then all nontrivial information for constructing the amplitude is contained in a single box numerator. More generally, at one loop whenever a $\mathrm{BCJ}$ representation of an $m$-point integrand is known, we can construct the entire amplitude starting from $m$-gon diagrams.

At one loop, there appear to be no difficulties finding numerators that obey global BCJ identities. Indeed, there are a variety of known examples that satisfy global identities in supersymmetric $[6,31]$ and nonsupersymmetric $[10,18]$ Yang-Mills theories. 




(a)

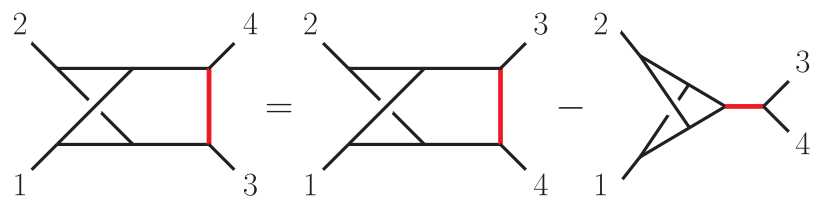

(b)

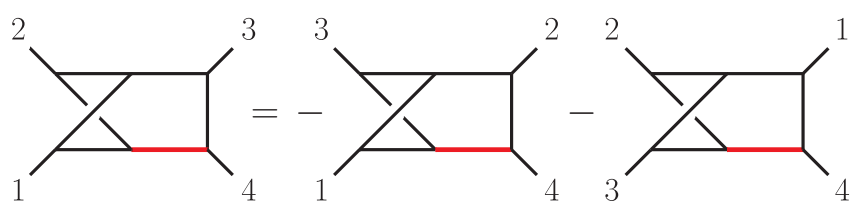

(c)

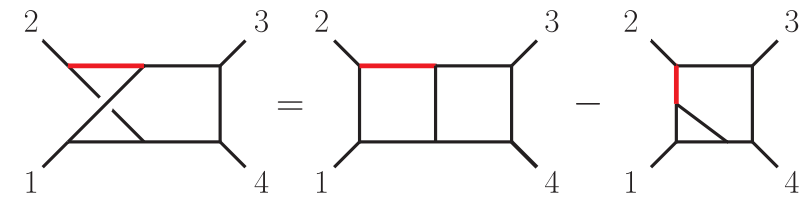

(d)

FIG. 3. Sample color and kinematic-numerator Jacobi relations for the two-loop four-point amplitudes involving (a) planar double-box diagrams and a box-triangle diagram, (b) nonplanar double-box diagrams and a nonplanar triangle diagram, (c) only nonplanar double-box diagrams and (d) planar and nonplanar double-box diagrams and a triangle-in-a-box diagram. The shaded (red) propagator indicates the line around which the Jacobi identities are applied.

At higher loops, the basic ideas are the same although the constructions rapidly become more difficult. Nevertheless, there are a variety of explicit nontrivial examples at two and higher loops where the global BCJ constraints are manifest $[2,5,6,18,32]$. Some examples of duality relations for the two-loop four-point amplitude are displayed in Fig. 3.

\section{BCJ duality on unitarity cuts}

As the number of loops or legs increases, it becomes more difficult to find expressions for amplitude integrands where global BCJ constraints are manifest. For example, constructing a representation of the five-loop four-point integrand of $\mathcal{N}=4$ super-Yang-Mills theory with manifest BCJ duality (2.2) remains a nontrivial challenge despite its central role for understanding the ultraviolet properties of gravity theories (see e.g. Refs. [17,20]). Similarly for two-loop four-point nonsupersymmetric Yang-Mills theory, as we shall see in Sec. IV, a minimal Ansatz with local numerators, manifest crossing symmetry and natural power-counting constraints cannot simultaneously satisfy global BCJ duality and unitarity.

The obvious strategy is to try to enlarge the Ansatz until a solution is found. However, for nontrivial cases, Ansätze can grow rapidly, making them impractical to work with. Here we take a different approach: We relax the BCJ duality constraints while keeping the essential doublecopy property, allowing us to obtain gravity integrands directly from gauge-theory ones. We find a form of the amplitude where the BCJ duality relations are manifest in a spanning set of unitarity cuts rather than on the full uncut integrands. (We note that manifest global BCJ duality in the gauge-theory integrand implies that the BCJ constraints are also valid on the spanning set of unitarity cuts; however, the converse is not true in general.) The doublecopy replacement rule of Eq. (2.4) then still holds. In a given color-dressed Yang-Mills unitarity cut, replacing the color factors by the corresponding duality-satisfying numerators ensures that the cut gauge-theory tree integrands composing the cut are properly converted to cut gravity tree integrands composing the corresponding gravity cut. If a gauge-theory numerator satisfies the duality in all cuts to which it contributes, then its double-copy gravity integrand will also satisfy these cuts and is therefore a valid gravity integrand. Importantly, the requirement that $\mathrm{BCJ}$ duality holds only on the cuts is less restrictive than having it hold globally in the integrands. This is true for two main reasons. Firstly, the on-shell conditions can remove contact contributions that violate a given BCJ numerator Jacobi relation. Secondly, on a given cut there are fewer relations because we include only those identities that act separately on trees composing the cuts. That is, we do not consider BCJ identities around a cut leg. It is still nontrivial, however, because we demand that the duality is manifest in all cuts of the integrand, which allows use of the double-copy replacement at the full integrand level.

As a simple first example, consider the one-loop fourpoint amplitude. A spanning set of unitarity cuts is the $s-$, $t$ - and $u$-channel versions of the two-particle cut illustrated in Fig. 4. After enforcing crossing symmetry, we only need to consider the $s$-channel cut. Now, instead of imposing the

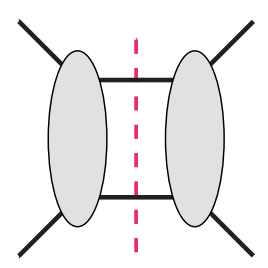

FIG. 4. Two-particle cut evaluated in all three channels determines one-loop four-point amplitudes. 
ZVI BERN, SCOTT DAVIES, and JOSH NOHLE
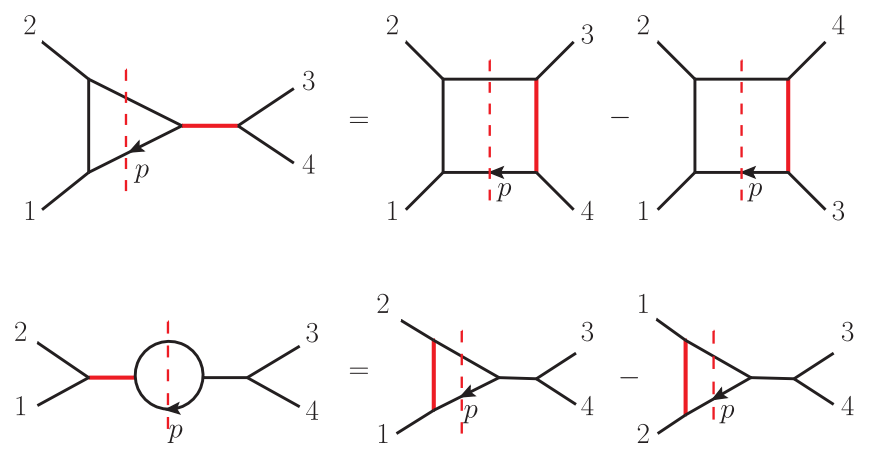

FIG. 5. The one-loop Jacobi relations with cut conditions imposed. The shaded (red) internal lines indicate the leg around which the Jacobi identities are applied. Internal legs intersected by the dashed lines are put on shell.

global BCJ conditions of Eq. (2.9), as illustrated in Fig. 5, we instead impose

$$
\begin{array}{r}
\left.\left(n_{12(34) ; p}^{(1)}-n_{1234 ; p}^{(1)}+n_{1243 ; p}^{(1)}\right)\right|_{\ell_{1}^{2}=\ell_{2}^{2}=0}=0, \\
\left.\left(n_{(12)(34) ; p}^{(1)}-n_{12(34) ; p}^{(1)}+n_{21(34) ; p}^{(1)}\right)\right|_{\ell_{1}^{2}=\ell_{2}^{2}=0}=0,
\end{array}
$$

where $\ell_{1}=p$ and $\ell_{2}=p-k_{1}-k_{2}$. Whereas Eq. (2.9) could be thought of as fully defining the triangle and bubble numerators in terms of the box-numerator master Ansatz, Eq. (2.10) should be thought of as constraint equations on separate Ansätze for the box, triangle and bubble numerators. Of course, for one-loop four-point amplitudes, there is not much point in imposing the relaxed $\mathrm{BCJ}$ conditions since no difficulties are encountered when imposing global BCJ constraints directly on the integrand. Indeed, there are a number of constructions of such integrands including the $D$-dimensional case with formal polarizations $[10,18,31]$.

At two loops, the basic idea is the same. Fig. 6 gives a spanning set of unitarity cuts which decomposes the integrand into sums of products of tree amplitudes. This set consists of the iterated two-particle cuts, (a) and (b), and the three-particle cut, (c). To impose the BCJ constraints on the cuts, we start with the BCJ identities on the numerators, as illustrated in Fig. 3. We then impose cut conditions as illustrated in Fig. 7. The figure shows a sample of BCJ relations in an iterated two-particle cut as well as in threeparticle cuts. Examples (a) and (b) in Fig. 7 are simply the BCJ identity in Fig. 3(d) but with on-shell conditions
PHYSICAL REVIEW D 93, 105015 (2016)

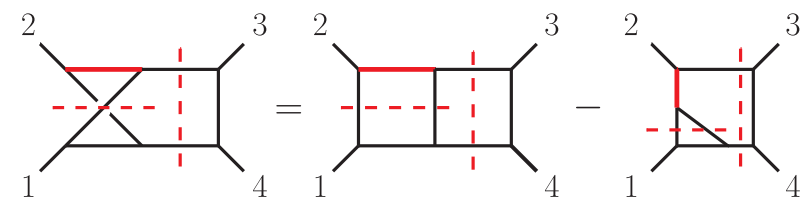

(a)

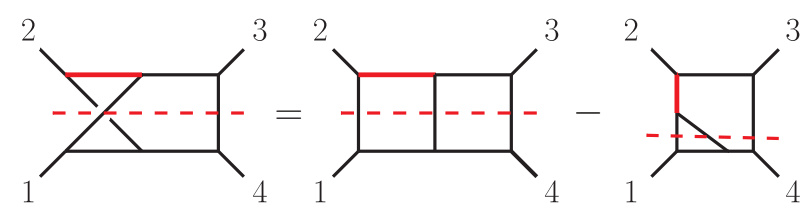

(b)

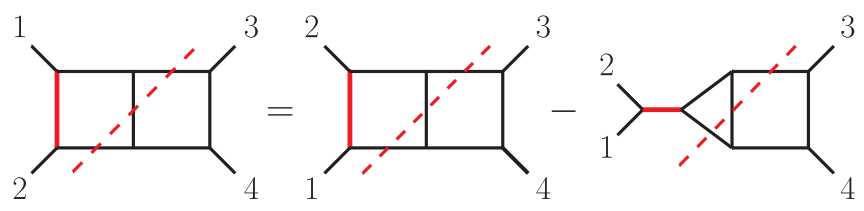

(c)

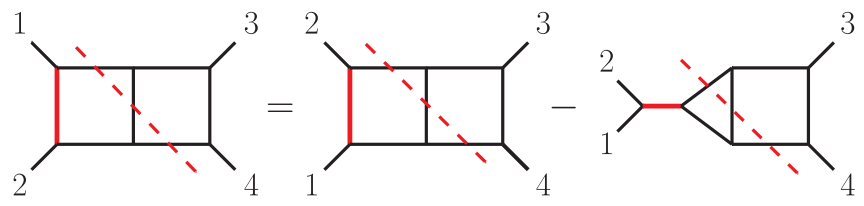

(d)

FIG. 7. Sample BCJ relations on cut two-loop diagrams. The diagrams represent kinematic numerators. Internal legs intersected by the dashed lines are put on shell. Examples (a) and (b) correspond to Fig. 3(d) with a horizontal-vertical two-particle cut applied and a three-particle cut applied, respectively. Examples (c) and (d) correspond to Fig. 3(a) with different three-particle cuts applied.

imposed on the cut legs. Examples (c) and (d) are slightly trickier because both are part of the same three-particle cut. The rule for grouping the cut diagrams into BCJ triplets is that the three cut diagrams are identical, including which legs are cut, except for the legs involving the BCJ duality. Again, the effect of the cut is to drop terms that cancel the cut propagators. This can help by allowing the use of a smaller Ansatz than would have otherwise been possible.

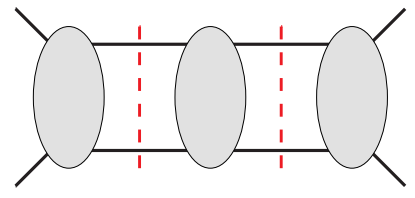

(a)

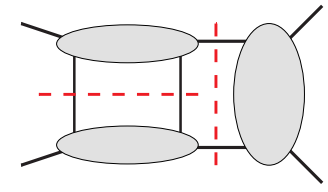

(b)



(c)

FIG. 6. A spanning set of generalized unitarity cuts for two-loop four-point color-dressed gauge-theory or gravity amplitudes, including (a) the "vertical-vertical" iterated two-particle cut, (b) the "horizontal-vertical" iterated two-particle cut and (c) the threeparticle cut. The exposed internal propagators are put on shell. 
One practical way to carry out this construction is to start with kinematic-numerator Ansätze with free parameters multiplying each possible term, subject to various desirable properties such as manifest locality, powercounting constraints and crossing symmetries. We then solve for some of the free parameters by matching to unitarity cuts-guaranteeing that the kinematic numerators will produce the correct Yang-Mills amplitude-and by imposing that BCJ duality holds on all of the cuts, as illustrated in Fig. 7. Assuming a consistent solution is found, the so-constructed gauge-theory integrands give double-copy gravity integrands, as desired, by replacing the color factors with corresponding numerator factors. Because the Yang-Mills integrands obey BCJ duality on each cut, the double-copy construction leads to gravity integrands that have the correct unitarity cuts. We then have correct gravity integrands, guaranteed by the $D$-dimensional unitarity method.

\section{BCJ NUMERATOR CONSTRUCTION: TWO-LOOP FOUR-POINT IDENTICAL HELICITY}

We consider nonsupersymmetric pure Yang-Mills theory defined by the usual Lagrangian,

$$
\mathcal{L}_{\mathrm{YM}}=-\frac{1}{4} F_{\mu \nu}^{a} F^{a \mu \nu},
$$

where $F_{\mu \nu}^{a}=\partial_{\mu} A_{\nu}^{a}-\partial_{\nu} A_{\mu}^{a}+g f^{a b c} A_{\mu}^{b} A_{\nu}^{c}$ is the field strength. The corresponding double-copy theory contains a graviton, dilaton and antisymmetric tensor field, as we discussed in Sec. II B. The Lagrangian for this theory is

$\mathcal{L}_{\mathrm{DC}}=\sqrt{-g}\left(-\frac{2}{\kappa^{2}} R+\frac{1}{2} \partial_{\mu} \phi \partial^{\mu} \phi+\frac{1}{6} e^{-2 \kappa \phi / \sqrt{D-2}} H_{\mu \nu \rho} H^{\mu \nu \rho}\right)$,

where $H_{\mu \nu \rho}=\partial_{\mu} A_{\nu \rho}+\partial_{\nu} A_{\rho \mu}+\partial_{\rho} A_{\mu \nu}$, and $A_{\mu \nu}=-A_{\nu \mu}$ is the rank-two antisymmetric tensor field. We use the mostly minus metric convention and the Ricci-curvature convention, $R_{\mu \nu} \equiv R_{\mu \alpha \nu}^{\alpha}$. Equation (3.2) corresponds to the lowenergy effective Lagrangian of the bosonic part of string theory. By using the double-copy construction, we reduce the problem of constructing integrands for amplitudes in the theory described by the Lagrangian (3.2) to constructing those for Yang-Mills theory (3.1). Pure gravity has been considered in Ref. [26]. The construction used there is different due to the need to introduce explicit physical-state

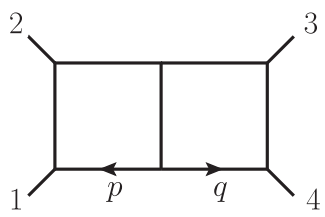

(a)

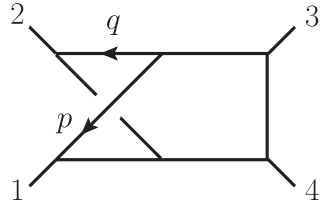

(b)
FIG. 8. The (a) planar and (b) nonplanar double-box integrals appearing in the identical-helicity pure Yang-Mills amplitude.

projectors in the unitarity cuts in order to remove the antisymmetric tensor and dilaton states from the theory. A possible route to applying the double-copy procedure to pure gravity was given in Ref. [3], where ghosts are used to cancel unwanted states.

As a warm-up for the two-loop four-point amplitude with arbitrary $D$-dimensional external states, we first look at the case of four-dimensional identical-helicity states. The identical-helicity pure Yang-Mills amplitude was first constructed in Ref. [19]. The construction relies on a version of the unitarity method that deals with external four-dimensional helicity states and internal states in $D$ dimensions, which is appropriate for dimensional regularization [8]. A full globally duality-satisfying representation is given in Ref. [18]. Here we discuss the original representation from Ref. [19] because it illustrates features we want: It does not satisfy global BCJ constraints, but it does satisfy the BCJ identities on the spanning set of cuts in Fig. 7 [1]. Therefore, replacing each color factor with a second copy of the corresponding kinematic numerator gives the integrand of the double-copy theory.

The two-loop all-plus helicity amplitude for pure YangMills theory from Ref. [19] is

$$
\begin{aligned}
\mathcal{A}_{4}^{(2)}\left(1^{+}, 2^{+}, 3^{+}, 4^{+}\right)= & -\frac{g^{6}}{4} \sum_{\mathcal{S}_{4}}\left(c_{1234}^{\mathrm{P}} \mathcal{I}^{\mathrm{P}}\left[n_{1234}^{\mathrm{P}}\right]\right. \\
& \left.+c_{12 ; 34}^{\mathrm{NP}} \mathcal{I}^{\mathrm{NP}}\left[n_{12 ; 34}^{\mathrm{NP}}\right]\right),
\end{aligned}
$$

where the sum runs over all 24 permutations of the external legs. The prefactor $1 / 4$ accounts for the overcount due to diagram symmetries. $c_{1234}^{\mathrm{P}}$ and $c_{12 ; 34}^{\mathrm{NP}}$ are the color factors obtained from the planar and nonplanar double-box diagrams shown in Fig. 8(a) and Fig. 8(b), respectively, by dressing each vertex with an $\tilde{f}^{a b c}$ and summing over the contracted color indices. $\mathcal{I}_{1234}^{\mathrm{P}}$ and $\mathcal{I}_{12 ; 34}^{\mathrm{NP}}$ are planar and nonplanar integrals given by

$$
\begin{aligned}
\mathcal{I}^{\mathrm{P}}\left[n_{1234}^{\mathrm{P}}\right] & =\int \frac{d^{D} p}{(2 \pi)^{D}} \frac{d^{D} q}{(2 \pi)^{D}} \frac{n_{1234}^{\mathrm{P}}}{p^{2} q^{2}(p+q)^{2}\left(p-k_{1}\right)^{2}\left(p-k_{1}-k_{2}\right)^{2}\left(q-k_{4}\right)^{2}\left(q-k_{3}-k_{4}\right)^{2},} \\
\mathcal{I}^{\mathrm{NP}}\left[n_{12 ; 34}^{\mathrm{NP}}\right] & =\int \frac{d^{D} p}{(2 \pi)^{D}} \frac{d^{D} q}{(2 \pi)^{D}} \frac{n_{12 ; 34}^{\mathrm{NP}}}{p^{2} q^{2}(p+q)^{2}\left(p-k_{1}\right)^{2}\left(q-k_{2}\right)^{2}\left(p+q+k_{3}\right)^{2}\left(p+q+k_{3}+k_{4}\right)^{2}},
\end{aligned}
$$

with the planar and nonplanar kinematic numerators, 


$$
\begin{aligned}
n_{1234}^{\mathrm{P}}= & -i \mathcal{T}\left[\frac{\left(D_{s}-2\right)^{2}}{s}(p+q)^{2} \lambda_{p}^{2} \lambda_{q}^{2}\left((p+q)^{2}+s\right)+16 s\left(\left(\lambda_{p} \cdot \lambda_{q}\right)^{2}-\lambda_{p}^{2} \lambda_{q}^{2}\right)\right. \\
& \left.+\left(D_{s}-2\right)\left(s\left(\lambda_{p}^{2} \lambda_{q}^{2}+\lambda_{p}^{2} \lambda_{p+q}^{2}+\lambda_{q}^{2} \lambda_{p+q}^{2}\right)+4(p+q)^{2}\left(\lambda_{p}^{2}+\lambda_{q}^{2}\right)\left(\lambda_{p} \cdot \lambda_{q}\right)\right)\right], \\
n_{12 ; 34}^{\mathrm{NP}}= & -i \mathcal{T} s\left[\left(D_{s}-2\right)\left(\lambda_{p}^{2} \lambda_{q}^{2}+\lambda_{p}^{2} \lambda_{p+q}^{2}+\lambda_{q}^{2} \lambda_{p+q}^{2}\right)+16\left(\left(\lambda_{p} \cdot \lambda_{q}\right)^{2}-\lambda_{p}^{2} \lambda_{q}^{2}\right)\right],
\end{aligned}
$$

where $\lambda_{p}, \lambda_{q}$ and $\lambda_{p+q}$ represent the $(-2 \epsilon)$-dimensional components of loop momenta $p, q$ and $(p+q)$, respectively. We take $D=4-2 \epsilon>4$ so that $\lambda_{p} \cdot k_{i}=0$, where the $k_{i}$ are four-dimensional external momenta. We have suppressed loop momentum labels for the numerators. The state-counting parameter is defined by $D_{s}=\delta^{\mu}{ }_{\mu}$ so that $D_{s}-2$ corresponds to the number of gluons states for each color circulating in the loop. The permutation-invariant kinematic prefactor is given by

$$
\mathcal{T} \equiv \frac{[12][34]}{\langle 12\rangle\langle 34\rangle},
$$

where $[i j]$ and $\langle i j\rangle$ are spinor products, defined in, for example, Ref. [33], and $s=\left(k_{1}+k_{2}\right)^{2}$ is a Mandelstam invariant. We have slightly rearranged the form of the amplitude given in Ref. [19] by absorbing the "bow-tie" contributions into the planar double box. With this choice the planar double-box numerator is nonlocal since it contains a term with a factor of $1 / s$. In this representation of the amplitude, all numerators of diagrams with topologies not matching either the planar or nonplanar doublebox diagram are taken to have vanishing numerators.

Now let us examine the issue of BCJ duality. As a first example, consider duality relation (a) in Fig. 3. Since all numerators except the planar and nonplanar double-box ones vanish, the duality relation reads

$$
n_{2134}^{\mathrm{P}}=n_{1234}^{\mathrm{P}} .
$$

In this case, the duality is satisfied trivially, even without any cut conditions imposed, because the planar numerator in Eq. (3.5) has a symmetry under the interchange of legs 1 and 2. The same holds for the nonplanar diagrams in BCJ relation (b) of Fig. 3. As a somewhat less trivial example, the third BCJ relation, (c), is also satisfied. This happens because the nonplanar numerator, $n_{12 ; 34}^{\mathrm{NP}}$ of Eq. (3.5), is independent of external labels except for the overall factor of $s$. Consequently, the BCJ relation is then

$$
n_{12 ; 34}^{\mathrm{NP}}+n_{13 ; 24}^{\mathrm{NP}}+n_{32 ; 14}^{\mathrm{NP}} \propto(s+t+u)=0,
$$

where $t=\left(k_{2}+k_{3}\right)^{2}$ and $u=\left(k_{1}+k_{3}\right)^{2}$ are the two other four-point Mandelstam invariants.

BCJ relation (d) in Fig. 3, however, does not hold if we do not impose cut conditions. Since the "triangle-in-box" numerator is zero in our representation, the relation reduces to a two-term identity,

$$
n_{12 ; 34}^{\mathrm{NP}}=n_{1234}^{\mathrm{P}} .
$$

This identity is obviously not satisfied by the numerators in Eq. (3.5) since the planar and nonplanar expressions are different. However, applying either the cut conditions in Fig. 7(a) or Fig. 7(b) discards all terms proportional to $(p+q)^{2}$, thereby removing discrepant terms in Eq. (3.9). [Note that for this relation we only need to consider the cut conditions of Fig. 7(a), (b) since the BCJ relation of Fig. 3(d) does not appear in cut (a) of Fig. 6.]

By systematically proceeding through all of the cuts in Fig. 6, it is straightforward to check that all BCJ identities hold for all diagrams composing each cut. This implies that a double-copy gravity integrand is obtained simply by replacing the color factors in Eq. (3.3) with a kinematic numerator and replacing the gauge-theory coupling with the gravitational one. Thus we obtain an expression for the identical-helicity two-loop four-graviton amplitude in the double-copy theory (3.2),

$$
\begin{aligned}
& \mathcal{M}_{4}^{(2)}\left(1^{+}, 2^{+}, 3^{+}, 4^{+}\right) \\
& =-i\left(\frac{\kappa}{2}\right)^{6} \frac{1}{4} \sum_{\mathcal{S}_{4}}\left(\mathcal{I}^{\mathrm{P}}\left[\left(n_{1234}^{\mathrm{P}}\right)^{2}\right]+\mathcal{I}^{\mathrm{NP}}\left[\left(n_{12 ; 34}^{\mathrm{NP}}\right)^{2}\right]\right) .
\end{aligned}
$$

The kinematic numerators are the squares of the gaugetheory ones in Eq. (3.5). We have directly confirmed that the spanning set of unitarity cuts (Fig. 6) of Eq. (3.10) are all correct, where the internal legs are taken to be in $D$ dimensions.

\section{BCJ NUMERATOR CONSTRUCTION: TWO-LOOP FOUR-POINT AMPLITUDE IN $D$ DIMENSIONS}

In this section, we describe the construction of the twoloop four-point double-copy gravity numerators with general formal polarization tensors in $D$ dimensions. This example demonstrates how one can sidestep difficulties when Ansätze are not sufficiently general to satisfy global BCJ duality. We do so by loosening the duality requirements to be manifest only on a spanning set of generalized unitarity cuts. Although the use of formal polarization vectors and tensors leads to much more complicated expressions than those for helicity amplitudes, it does have the advantage that formal polarization amplitudes are valid in all dimensions, and the more straightforward 
conventional dimensional-regularization scheme [34] can be used instead of the four-dimensional-helicity scheme [35]. They are also useful for studying evanescent effects in gravity, which can be quite subtle [26].

\section{A. Constraining an Ansatz}

The construction begins in pure Yang-Mills theory. Our goal is to find a representation of the pure Yang-Mills twoloop four-point amplitude of the form given in Eq. (2.6) that has the double-copy property, allowing us to immediately obtain the corresponding gravity amplitude in the form of Eq. (2.7).

To start, we build an Ansatz for the kinematic numerators with the following properties imposed:

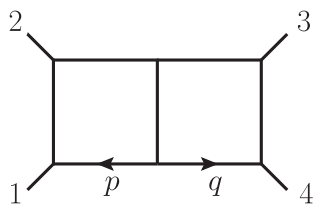

(1)

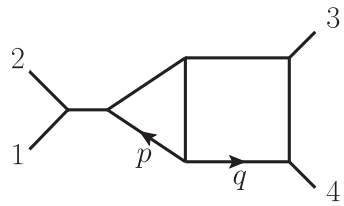

$(4)$

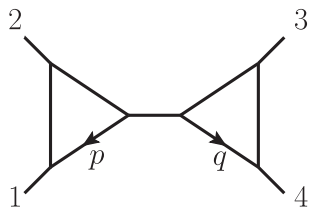

(7)

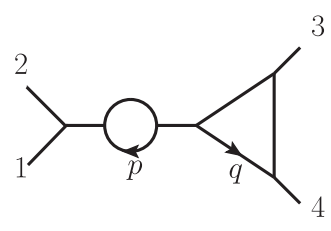

(10)

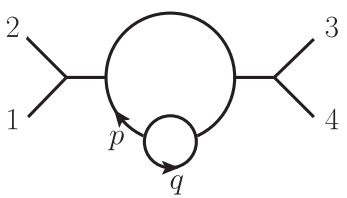

(13)

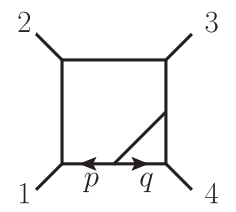

(2)

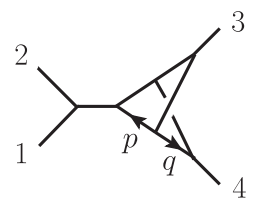

(5)

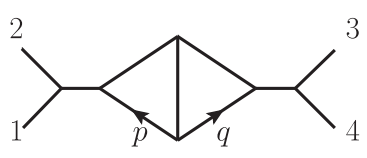

(8)

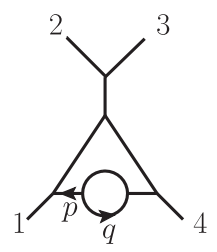

(11)

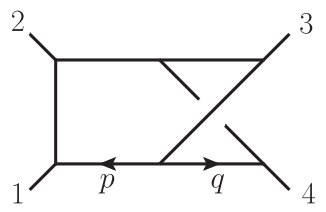

(3)

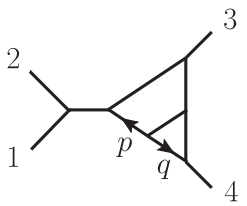

(6)

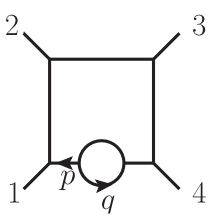

(9)

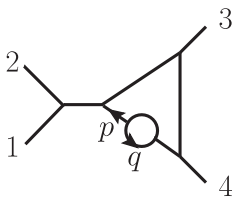

(12)

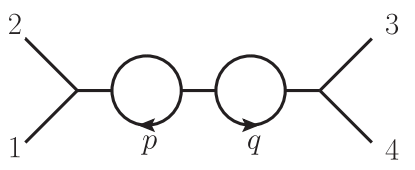

(14)

FIG. 9. Diagrams (1)-(14) are the relevant two-loop four-point diagrams with only cubic vertices. All other diagrams in the amplitude are obtained by relabeling external legs. Tadpole and bubble-on-external-leg diagrams, which integrate to zero, are not included. 
diagram's numerator simply by relabeling momenta, polarizations and color factors.

(5) Diagram symmetries respected. The numerator should reflect the symmetries of the diagrams. For instance, if the color factors change by sign under the symmetry, then the numerator should also change by the same sign.

(6) Unitarity cuts. The cuts of the Ansatz should correctly match a spanning set of unitarity cuts. This is simply a requirement that the Ansatz be a correct representation of the Yang-Mills amplitude.

(7) BCJ double-copy property. The double-copy property (2.4) should hold, allowing us to obtain amplitudes in the double-copy theory from gauge-theory ones simply by replacing color factors with kinematic numerators.

Except for properties 3 and 7, these are standard properties that ordinary Feynman diagrams in Feynman gauge possess. The ghost contributions of Feynman diagrams ensure that physical-state projectors are properly reconstructed across unitarity cuts, but they do so in a way that maintains the manifest locality of the numerators. We are of course seeking a representation of the amplitude with all of these properties as well as additional ones, in particular the nontrivial final property 7 that the double-copy property holds. Note that we have not imposed that the global BCJ identities hold manifestly, only that the weaker double-copy property holds.

For the two-loop four-point amplitude, the set of diagrams with only cubic vertices is given by the independent relabelings of the diagrams in Fig. 9. As usual, tadpoles have a vanishing color factor, and bubble-on-external-leg contributions are dropped because they integrate to zero in dimensional regularization through a cancellation of ultraviolet and infrared singularities. (However, one needs to be aware of this cancellation when trying to extract only an ultraviolet divergence or an infrared divergence from the amplitude.)

We start by imposing the first five conditions on the Ansatz. Each diagram numerator Ansatz, $n_{j}$, is a linear combination of monomials, $M_{j k}$, subject to the powercounting constraint in property 2 above:

$$
n_{j}=\sum_{k} a_{j k} M_{j k}
$$

where $a_{j k}$ are the undetermined coefficients of the Ansatz for diagram $(j)$. The monomials are built out of independent dot products,

$$
\begin{array}{lllll}
\varepsilon_{i} \cdot \varepsilon_{j}, & \varepsilon_{i} \cdot k_{j}, & \varepsilon_{i} \cdot p, \quad \varepsilon_{i} \cdot q, & k_{i} \cdot p, \\
k_{i} \cdot q, & p^{2}, & q^{2}, \quad p \cdot q, \quad s, & t,
\end{array}
$$

with leg labels $i, j=1,2,3,4$. We only include monomials that are independent under momentum-conservation, on-shell and transversality conditions:

$$
k_{4}=-k_{1}-k_{2}-k_{3}, \quad k_{i}^{2}=0, \quad k_{i} \cdot \varepsilon_{i}=0 .
$$

By imposing constraints 1-4, we obtain an Ansatz with 9814 terms for diagram (1), 9452 terms for diagram (2), 9902 terms for diagram (3) and so on. Due to property 5, many terms are related by diagram symmetries. Imposing these symmetry relations reduces the number of undetermined coefficients respectively to $2703,4748,2546$ and so on for these diagrams.

\section{B. Global BCJ identities on integrand}

Can we consistently impose the global BCJ identities with the above constraints? If we could, then this would be an efficient route to constructing the loop integrand. In attempting this, we follow the strategy described in detail in Ref. [5]. By imposing global BCJ identities, we are strengthening constraint 7 to

( $\left.7^{\prime}\right)$ BCJ duality manifest in integrand. We demand that the diagram numerators obey the full set of global dual Jacobi relations in Eq. (2.2).

This constraint allows us to express all numerators in terms of two master diagrams. For the two-loop four-point amplitudes, we choose the masters to be diagrams (1) and (2) in Fig. 9. These are then the only diagrams that require an Ansatz, and the values of the remaining diagrams are generated by the $\mathrm{BCJ}$ numerator relations. This gives us an Ansatz for the entire amplitude. As mentioned, the two master diagrams start with 9814 and 9452 terms, respectively, where each term is multiplied by a free parameter. With the first five constraints and global BCJ constraints imposed, we are reduced to 1279 total free parameters, noting that diagram-symmetry constraints fix a large number of coefficients. We then systematically step through and impose the spanning set of unitarity cuts in Fig. 6.

The standard way to evaluate the cuts is to sew together tree amplitudes appearing in the cuts using physical-state projectors. The $D$-dimensional projectors introduce lightcone denominators, which must cancel away in the final gauge-invariant cut expressions. We avoid introducing such spurious denominators by using ghost fields via Feynman diagrams. While a Feynman-diagram representation is not particularly enlightening or compact, it gives a simple means to generate target expressions for checking unitarity cuts. The cuts of ordinary Feynman-gauge Feynman diagrams automatically contain only the usual Feynman propagators and therefore never introduce spurious denominators in the first place. In any case, the unitarity cuts are gauge invariant, and their values do not depend on how they are generated.

Once target expressions for the cuts are generated, the next step is to fix some or all of the remaining parameters in the Ansatz by matching its cuts to the target cuts. After some effort, we find that the result of this procedure is that the constraints 1-6 listed above are incompatible with 
finding a solution to the global BCJ identities. We can consistently impose unitarity cuts (b) and (c) of Fig. 6, but the iterated two-particle cut (a) by itself is inconsistent with conditions 1-6 and $7^{\prime}$. We can localize the problem by observing that unitarity cuts (b) and (c) of Fig. 6 are simultaneously consistent and fix all relevant contact terms in the Ansatz except those proportional to $(p+q)^{2}$, corresponding to the labels in Fig. 9. Hence, the contact terms involving the middle propagator of diagrams (1), (4) and (8) in Fig. 9 cannot be consistently constrained. [We directly confirmed that this is the source of the problem by using a cut-merging procedure [36] through which we added terms to these diagrams proportional to $(p+q)^{2}$, making them consistent with unitarity at the cost of losing the double-copy property.]

One could hope to conquer this obstacle by relaxing some or all of constraints 1-4. In simple cases, this is usually the best strategy. However, the minimal Ansatz for each diagram numerator is already fairly complicated, and the complexity grows rapidly as these constraints are released. This becomes more problematic as the number of loops increases. The difficulty here in finding an Ansatz compatible with both unitarity and global BCJ constraints is a simpler version of difficulties encountered at five loops in $\mathcal{N}=4$ super-Yang-Mills theory [17].

\section{BCJ duality on generalized unitarity cuts}

Here we explore an alternative tactic of preserving constraints $1-5$ that keeps the complexity of each numerator Ansatz under control, but instead releases the constraint that global BCJ duality is manifest in the integrand. We do so in such a way that preserves the crucial double-copy property that allows us to obtain gravity integrands from gauge-theory ones. This strategy amounts to a trade-off between maintaining the relative simplicity of minimal Ansätze and losing the global BCJ relations that allow us to write the full amplitude in terms of master diagrams.

In order to maintain the double-copy property 7 , we impose instead the following condition:

( 7 ") BCJ duality on generalized unitarity cuts. Demand that BCJ duality is manifest in a spanning set of generalized unitarity cuts of the amplitude.

This effectively means that the cuts of amplitudes are expressed in terms of sums of products of tree amplitudes, where each tree is in a form where BCJ duality is manifest. Tree amplitudes can always be put into a BCJ-satisfying form, but what makes this condition nontrivial is the requirement of a single integrand having the property that for each cut in a spanning set of cuts, BCJ duality is manifest without needing rearrangements. This then guarantees that under the replacement (2.4) in the integrand, the cuts match those of the double-copy gravity theory. It is however a weaker condition than requiring that $\mathrm{BCJ}$ duality be manifest globally in the integrand itself.
To find an integrand with the desired properties, we use a distinct Ansatz for each diagram in Fig. 9. Applying conditions 1-4 above, we start with a rather large Ansatz containing 120904 parameters. This is reduced to 28204 free parameters by condition 5 , which requires that the numerators respect the diagram symmetries.

As in Sec. IV B, we compare each unitarity cut against the target cuts. In addition, we impose that the numerators of the cut diagrams obey all BCJ relations, as illustrated in the examples of Fig. 7. In this case, there is no problem finding a solution to both the BCJ relations and the spanning set of cuts in Fig. 6. In fact, the final solution has 6322 free parameters. These free parameters do not alter any unitarity cuts, but merely move contact terms between diagrams while maintaining BCJ duality on the spanning set of cuts in Fig. 6.

The solution is lengthy and is found in the Supplemental Material [37]. ${ }^{1}$ Its length is largely due to the fact that we use formal polarization vectors, which allows us the generality to work in $D$ dimensions. In the Supplemental Material [37], numerators.m, the vertices, inverse propagators, numerators, color factors and symmetry factors of each diagram in Fig. 9 are given in Mathematica syntax as a list. In this syntax, the information of diagram $(j)$ needed for Eqs. (2.6) and (2.7) can be accessed by vertices[[j]], propagators[[j]], numerators[[j]], color[[j]] and symmetry [[j]], where "[[j]]" takes the $j$ th entry in the list. For example, the planar double box, diagram (1), is accessed by vertices[[1]], which returns

$$
\begin{gathered}
\{\{-1\},\{-2\},\{-3\},\{-4\},\{1,9,-5\},\{2,-7,-9\}, \\
\{3,-11,-8\},\{4,-6,11\},\{5,10,6\},\{7,8,-10\}\} .
\end{gathered}
$$

The structure of vertices[[j]] is such that a one-element list represents an external momentum label, a threeelement list represents a cubic vertex in clockwise ordering, and a positive (negative) number represents an outgoing (incoming) momentum from (to) the vertex. These labels match those displayed in Fig. 10 for the planar double box.

Considering diagram (1) as an example, the inverse propagators, denoted $\prod_{\alpha_{1}} p_{\alpha_{1}}^{2}$ in Eqs. (2.6) and (2.7), are accessed by propagators[[1]], which returns

$$
\begin{aligned}
& \left.(\mathrm{k}[1]-\mathrm{k}[5])^{\wedge} 2 *(\mathrm{k}[1]+\mathrm{k}[2]-\mathrm{k}[5])^{\wedge} 2 * \mathrm{k}[5] \wedge 2 * \mathrm{k}[6]\right]^{\wedge} 2 * \\
& (\mathrm{k}[1]+\mathrm{k}[2]+\mathrm{k}[6])^{\wedge} 2 *(\mathrm{k}[1]+\mathrm{k}[2]+\mathrm{k}[3]+\mathrm{k}[6])^{\wedge} 2 * \\
& (\mathrm{k}[5]+\mathrm{k}[6])^{\wedge} 2 .
\end{aligned}
$$

\footnotetext{
${ }^{1}$ The remaining 6322 free coefficients are set to zero in this file. It would be interesting to see if there are other useful properties that could be enforced with wise choices of the free parameters.
} 


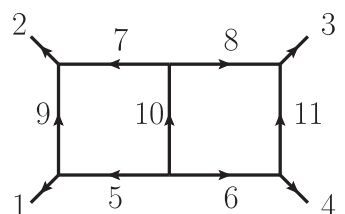

FIG. 10. A diagram with labels corresponding to those of the Supplemental Material [37]. The arrows give the direction of the momenta.

These of course follow from momentum conservation. The corresponding numerator for diagram (1), $n_{1}$, is accessed by numerators[[1]], which returns

$(\mathrm{I} / 189) *(-140278+24959 * \mathrm{Ds}) * \mathrm{ke}[1,2] * \mathrm{ke}[2,1] *$

$\operatorname{ke}[2,3] * \operatorname{ke}[2,4] * \operatorname{kk}[1,2]+\cdots$,

where the "..." signifies 8223 additional terms found in the Supplemental Material [37]. Note that we group factors of $i$ coming from the Feynman propagators with the numerator. Also, take note of the overall prefactors in Eqs. (2.6) and (2.7). The parameter Ds is a state-counting parameter that comes from the contraction of metric tensors. It is the same parameter as $D_{s}$ in Eq. (3.5). Depending on the regularization scheme, in four dimensions it can be Ds $=4$ or Ds $=4-2 \epsilon$.

The color factor can be read off directly from the vertices by replacing each triplet of numbers in the vertices $\{i, j, k\}$ with an $\tilde{f}^{a_{i} a_{j} a_{k}}$ (dropping the index signs). For example, color[[1]] returns

$$
\begin{aligned}
& \operatorname{tf}[1,9,5] * \operatorname{tf}[2,7,9] * \operatorname{tf}[3,11,8] * \\
& \operatorname{tf}[4,6,11] * \operatorname{tf}[5,10,6] * \operatorname{tf}[7,8,10],
\end{aligned}
$$

corresponding to the diagram (1) color factor,

$$
c_{1}=\tilde{f}^{a_{1} a_{9} a_{5}} \tilde{f}^{a_{2} a_{7} a_{9}} \tilde{f}^{a_{3} a_{11} a_{8}} \tilde{f}^{a_{4} a_{6} a_{11}} \tilde{f}^{a_{5} a_{10} a_{6}} \tilde{f}^{a_{7} a_{8} a_{10}} .
$$

Finally, the symmetry factor $S_{1}$ for diagram (1), for instance, is given by symmetry[[1]], which returns 4 .

Taking the numerators, propagators, color and symmetry factors in the Supplemental Material [37] and inserting them into Eq. (2.6) gives the two-loop four-point YangMills amplitude. Similarly, the corresponding double-copy gravity amplitude is obtained by inserting the appropriate factors into Eq. (2.7). We have explicitly checked that the constructed gauge and gravity amplitudes correctly satisfy the spanning set of generalized unitarity cuts given in Fig. 6. In the gravity amplitude verification, the integrands were constructed in two different ways as a cross check: The primary way uses two copies of the gauge-theory numerators from the Supplemental Material [37], whereas the second way uses one copy from the Supplemental Material [37] and the other copy from numerators generated by nonsupersymmetric Yang-Mills theory Feynman-gauge Feynman rules. Both ways give correct gravity results compatible with the unitarity constraints.

\section{ULTRAVIOLET PROPERTIES}

In this short section, we summarize results on the ultraviolet singularities derived from the identical-helicity four-graviton amplitude discussed in Sec. III. The counterterm is the well-studied two-loop $R^{3}$ counterterm [26,38]. These results summarize those of Ref. [18] for the bare contribution and Refs. [26] and [39] for the subdivergence subtractions.

The result for the two-loop bare divergence is already given in Ref. [18]. That paper uses a different representation of the integrand, where global BCJ relations hold between the diagrams. In any case, after integrating we obtain the same bare ultraviolet divergence. Integral tables may be found in the appendices of Ref. [18]. Using dimensional regularization, the infrared and ultraviolet divergences are mixed together. However, the infrared singularities are simple and known ahead of time [40], so they are easily subtracted out. (Ref. [18] also used an alternate method based on introducing a mass regulator; the results for the ultraviolet divergences are the same for either method.) The net result is that the bare two-loop divergence is

$$
\left.\mathcal{M}^{(2)}\left(1^{+}, 2^{+}, 3^{+}, 4^{+}\right)\right|_{\text {bare UV div }}=\frac{1}{\epsilon} \frac{83}{2700} \mathcal{K},
$$

where

$$
\mathcal{K} \equiv\left(\frac{\kappa}{2}\right)^{6} \frac{i}{(4 \pi)^{4}} \operatorname{stu}\left(\frac{[12][34]}{\langle 12\rangle\langle 34\rangle}\right)^{2}
$$

However, as discussed in Ref. [26], an important subtlety is that there are also subdivergence subtractions even though there are no corresponding one-loop divergences in $D=4$. The double-copy theory does have one-loop divergences $[18,41]$, but these divergences are in four-matter amplitudes which cannot appear as subdivergences in the two-loop fourgraviton amplitude. The origin of this curious behavior is evanescent operators, which can act as counterterms. The Gauss-Bonnet term is one such evanescent operator. In the double-copy theory, the Gauss-Bonnet counterterm is

$\mathcal{L}_{\mathrm{GB}}^{\mathrm{CT}}=-\frac{1}{(4 \pi)^{2}}\left(\frac{53}{90}+\frac{1}{360}+\frac{91}{360}\right) \sqrt{-g}\left(R^{2}-4 R_{\mu \nu}^{2}+R_{\mu \nu \rho \sigma}^{2}\right)$,

where the three numbers in the parenthesis correspond to the contributions from the graviton, dilaton and antisymmetric tensor, respectively. The coefficients are proportional to the ones that appear in the trace anomaly [42]. The GaussBonnet theorem implies that in four dimensions, the operator 
(5.3) is a total derivative. When antisymmetric tensors are coupled to gravity, another relevant one-loop four-point divergence is that of two gravitons and two antisymmetric tensors, which has a counterterm,

$$
\mathcal{L}_{R H H}^{\mathrm{CT}}=-\frac{5}{3}\left(\frac{\kappa}{2}\right)^{2} \frac{1}{(4 \pi)^{2}} \frac{1}{\epsilon} \sqrt{-g} R_{\rho \sigma}^{\mu \nu} H_{\mu \nu \alpha} H^{\alpha \rho \sigma} .
$$

Like the Gauss-Bonnet counterterm (5.3), this operator is evanescent. In $D=4$, we can dualize the antisymmetric tensors to scalars, which collapses the Riemann tensor into the Ricci scalar and tensor. Under field redefinitions, they can then be removed in favor of the dualized scalars, eliminating the one-loop divergence in two-graviton twoantisymmetric-tensor amplitudes when the external states are in $D=4$.

As discussed in Ref. [26], there are two types of counterterm subtractions, as illustrated by representative diagrams in Fig. 11. The single-counterterm insertion subdivergence corresponding to Fig. 11(a) is [39]

$$
\left.\mathcal{M}^{(2)}\left(1^{+}, 2^{+}, 3^{+}, 4^{+}\right)\right|_{\text {single CT }}=\frac{1}{\epsilon}\left(-\frac{6004}{675}+\frac{25}{3}\right) \mathcal{K},
$$

where the first number is the contribution from the Gauss-Bonnet counterterm in Eq. (5.3) and the second is the contribution from the $R H H$ counterterm in Eq. (5.4). In addition, there is the double Gauss-Bonnet counterterm insertion illustrated by a representative diagram in Fig. 11(b). This is given by

$$
\left.\mathcal{M}^{(2)}\left(1^{+}, 2^{+}, 3^{+}, 4^{+}\right)\right|_{\text {double CT }}=\frac{1}{\epsilon} \frac{11552}{675} \mathcal{K} .
$$

Adding together the three contributions in Eqs. (5.1), (5.5) and (5.6) gives the total two-loop divergence,

$$
\left.\mathcal{M}^{(2)}\left(1^{+}, 2^{+}, 3^{+}, 4^{+}\right)\right|_{\text {total }}=\frac{1}{\epsilon} \frac{199}{12} \mathcal{K}
$$

This divergence can be removed from the amplitude by adding an $R^{3}$ counterterm to the theory,

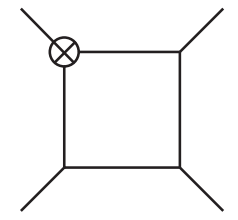

(a)

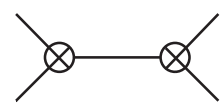

(b)
FIG. 11. Representative diagrams of the (a) single-counterterm and (b) double-counterterm insertions.

$$
\mathcal{L}_{R^{3}}^{\mathrm{CT}}=\frac{199}{720}\left(\frac{\kappa}{2}\right)^{2} \frac{1}{(4 \pi)^{4}} \frac{1}{\epsilon} \sqrt{-g} R_{\gamma \delta}^{\alpha \beta} R_{\rho \sigma}^{\gamma \delta} R_{\alpha \beta}^{\rho \sigma} .
$$

Up to the coefficient, this is the same divergence that appears in pure gravity at two loops [26,38]. As already noted, the double-copy theory also has a one-loop divergence in the matter sector [41].

As discussed in Ref. [26], the divergence itself is not physical and is modified by duality transformations. However, the renormalization scale is unaltered. The coefficient of the $\ln \mu^{2}$ dependence is easily extracted by noting that, for the bare two-loop part, the $\ln \mu^{2}$ coefficient is twice the coefficient of the $1 / \epsilon$ divergence, for the single counterterm, it is equal to the $1 / \epsilon$ coefficient, and for the double-insertion tree contribution, it vanishes. This follows from dimensional analysis of the loop integrals, with measure $\int d^{4-2 \epsilon} \ell$ per loop, which requires an overall factor of $\mu^{2 L \epsilon}$ at $L$ loops. The counterterm subtractions themselves are pure poles which do not carry such factors. This then gives the $\ln \mu^{2}$ dependence,

$$
\left.\mathcal{M}_{4}^{2 \text {-loop }}\left(1^{+}, 2^{+}, 3^{+}, 4^{+}\right)\right|_{\ln \mu^{2}}=-\mathcal{K} \frac{1}{2} \ln \mu^{2},
$$

in agreement with the general formula given in Ref. [26],

$\left.\mathcal{M}_{4}^{2 \text {-loop }}\left(1^{+}, 2^{+}, 3^{+}, 4^{+}\right)\right|_{\ln \mu^{2}}=-\mathcal{K} \frac{N_{b}-N_{f}}{8} \ln \mu^{2}$,

where $N_{b}$ is the number of bosonic states and $N_{f}$ is the number of fermionic states.

Reference [39] will provide further details on a variety of theories, including the double-copy gravity theory (3.2), as well as present full amplitudes including their finite parts. It is an interesting open problem to extract the divergences from the amplitude in terms of formal polarization tensors to ensure the consistency for all helicity configurations. However, one encounters high-rank tensor double-box integrals making the integration nontrivial.

\section{CONCLUSIONS}

Constructions of higher-loop amplitudes in supergravity have provided a wealth of new nontrivial information on gravity theories. This includes tantalizing new effects such as enhanced ultraviolet cancellations [20] and dependence of leading divergences on evanescent effects and duality transformations $[26,39]$. In addition, the only two known divergences in four dimensions in pure Einstein gravity and pure ungauged supergravity theories display anomaly-like behavior, as discussed in Refs. [24,26].

To further explore these effects and to uncover new ones, we need more efficient ways to obtain gravity amplitudes at high loop orders. At present the most powerful means for doing so is based on the duality between color and kinematics $[1,2]$ in conjunction with the unitarity method 
[7]. However, at sufficiently high loop orders or in complicated cases with little or no supersymmetry, it can be nontrivial to find representations of the amplitude where the duality is manifest. It may be that Ansätze that are sufficiently general to be compatible with both unitarity cuts and global BCJ constraints are impractical to work with. The strategy presented here for dealing with such difficulties is to loosen the BCJ constraints by demanding that they hold manifestly only on unitarity cuts instead of on uncut integrands. This allows us to use simpler Ansätze for individual diagrams yet retain the key double-copy property. The cost is that we lose the ability to determine the gauge-theory integrand from a small number of master diagrams.

After warming up on the case of identical helicities, we demonstrated our strategy in action on the two-loop four-point pure Yang-Mills amplitude with $D$-dimensional external polarizations. We first showed that a minimal Ansatz, where locality, crossing symmetry and Feynmandiagram-like power counting are manifest is not compatible simultaneously with both global BCJ duality on the integrand and unitarity. On the other hand, no difficulties are encountered when the duality requirements are loosened so that they are manifest only on a spanning set of generalized unitarity cuts. The so-constructed Yang-Mills integrand immediately produces a corresponding gravity integrand via the double-copy procedure.
Using results from Refs. [18,26,39], we extracted the two-loop ultraviolet divergence from the identical-helicity amplitude of the double-copy gravity theory. As explained in Ref. [26], in contrast to the divergence itself, the renormalization-scale dependence follows a universal formula that depends only on the number of four-dimensional bosonic and fermionic states and not on their spin. Further details will be given in Ref. [39]. It would be interesting to carry out the same analysis on the case of general polarizations using conventional dimensional regularization to confirm the generality of the results.

We hope that the strategy presented here will help lead to new constructions of multiloop (super)gravity amplitudes, and we look forward to the new insights that they will provide.

\section{ACKNOWLEDGMENTS}

We thank John Joseph Carrasco, Clifford Cheung, HuanHang Chi, Tristan Dennen, Lance Dixon, Henrik Johansson and Radu Roiban for many interesting and useful discussions and collaboration on related work. This material is based upon work supported by the Department of Energy under Award No. DE-SC0009937. Z. B. is grateful to the Simons Foundation for support. We gratefully acknowledge Mani Bhaumik for his continued generous support.
[1] Z. Bern, J. J. M. Carrasco, and H. Johansson, New relations for gauge-theory amplitudes, Phys. Rev. D 78, 085011 (2008).

[2] Z. Bern, J. J. M. Carrasco, and H. Johansson, Perturbative Quantum Gravity as a Double Copy of Gauge Theory, Phys. Rev. Lett. 105, 061602 (2010).

[3] H. Johansson and A. Ochirov, Pure gravities via colorkinematics duality for fundamental Matter, J. High Energy Phys. 11 (2015) 046.

[4] H. Johansson and A. Ochirov, Color-kinematics duality for QCD amplitudes, arXiv:1507.00332; L. de la Cruz, A. Kniss, and S. Weinzierl, Proof of the fundamental BCJ relations for QCD amplitudes, J. High Energy Phys. 09 (2015) 197.

[5] Z. Bern, J. J. M. Carrasco, L. J. Dixon, H. Johansson, and R. Roiban, Simplifying multiloop integrands and ultraviolet divergences of gauge theory and gravity amplitudes, Phys. Rev. D 85, 105014 (2012).

[6] J. J. Carrasco and H. Johansson, Five-point amplitudes in $N=4$ super-Yang-Mills theory, Phys. Rev. D 85, 025006 (2012).

[7] Z. Bern, L. J. Dixon, D. C. Dunbar, and D. A. Kosower, One loop $n$ point gauge theory amplitudes, unitarity, and collinear limits, Nucl. Phys. B425, 217 (1994); Fusing gauge theory tree amplitudes into loop amplitudes, Nucl. Phys. B435, 59 (1995); Z. Bern, L. J. Dixon, D. C. Dunbar, M. Perelstein, and J.S. Rozowsky, On the relationship between Yang-Mills theory and gravity and its implication for ultraviolet divergences, Nucl. Phys. B530, 401 (1998); Z. Bern, J. J. M. Carrasco, H. Johansson, and D. A. Kosower, Maximally supersymmetric planar YangMills amplitudes at five loops, Phys. Rev. D 76, 125020 (2007).

[8] Z. Bern and A. G. Morgan, Massive loop amplitudes from unitarity, Nucl. Phys. B467, 479 (1996); Z. Bern, L. J. Dixon, and D. A. Kosower, Progress in one loop QCD computations, Annu. Rev. Nucl. Part. Sci. 46, 109 (1996).

[9] R. Monteiro and D. O'Connell, The kinematic algebra from the self-dual sector, J. High Energy Phys. 07 (2011) 007; N. E. J. Bjerrum-Bohr, P. H. Damgaard, R. Monteiro, and D. O'Connell, Algebras for amplitudes, J. High Energy Phys. 06 (2012) 061; R. Monteiro and D. O'Connell, The kinematic algebras from the scattering equations, J. High Energy Phys. 03 (2014) 110.

[10] J. Nohle, Color-kinematics duality in one-loop four-gluon amplitudes with matter, Phys. Rev. D 90, 025020 (2014). 
[11] S. He, R. Monteiro, and O. Schlotterer, String-inspired BCJ numerators for one-loop MHV amplitudes, arXiv: 1507.06288 .

[12] R. H. Boels, B. A. Kniehl, O. V. Tarasov, and G. Yang, Color-kinematic duality for form factors, J. High Energy Phys. 02 (2013) 063; S. Badger, G. Mogull, A. Ochirov, and D. O'Connell, A complete two-loop, five-gluon helicity amplitude in Yang-Mills theory, J. High Energy Phys. 10 (2015) 064.

[13] R. Saotome and R. Akhoury, Relationship Between Gravity and Gauge Scattering in the High Energy Limit, J. High Energy Phys. 01 (2013) 123; R. Monteiro, D. O'Connell, and C. D. White, Black holes and the double copy, J. High Energy Phys. 12 (2014) 056; A. Luna, R. Monteiro, D. O'Connell, and C. D. White, The classical double copy for Taub-NUT spacetime, Phys. Lett. B 750, 272 (2015).

[14] T. Bargheer, S. He, and T. McLoughlin, New Relations for Three-Dimensional Supersymmetric Scattering Amplitudes, Phys. Rev. Lett. 108, 231601 (2012); J. Broedel and L. J. Dixon, Color-kinematics duality and double-copy construction for amplitudes from higher-dimension operators, J. High Energy Phys. 10 (2012) 091; Y.t. Huang and H. Johansson, Equivalent $D=3$ Supergravity Amplitudes from Double Copies of Three-Algebra and Two-Algebra Gauge Theories, Phys. Rev. Lett. 110, 171601 (2013); M. Chiodaroli, Q. Jin, and R. Roiban, Color/kinematics duality for general Abelian orbifolds of $N=4$ super Yang-Mills theory, J. High Energy Phys. 01 (2014) 152; M. Chiodaroli, M. Günaydin, H. Johansson, and R. Roiban, Scattering amplitudes in $\mathcal{N}=2$ Maxwell-Einstein and Yang-Mills/ Einstein supergravity, J. High Energy Phys. 01 (2015) 081; A. Ochirov and P. Tourkine, BCJ duality and double copy in the closed string sector, J. High Energy Phys. 05 (2014) 136.

[15] S. Oxburgh and C. D. White, BCJ duality and the double copy in the soft limit, J. High Energy Phys. 02 (2013) 127; Y. J. Du, B. Feng, and C. H. Fu, The construction of dual-trace factor in Yang-Mills theory, J. High Energy Phys. 07 (2013) 057; C. H. Fu, Y. J. Du, and B. Feng, Note on construction of dual-trace factor in Yang-Mills theory, J. High Energy Phys. 10 (2013) 069; M. Tolotti and S. Weinzierl, Construction of an effective Yang-Mills Lagrangian with manifest BCJ duality, J. High Energy Phys. 07 (2013) 111; H. Johansson, A. Sabio Vera, E. Serna Campillo, and M. A. Vázquez-Mozo, Color-kinematics duality in multi-Regge kinematics and dimensional reduction, J. High Energy Phys. 10 (2013) 215; A. Anastasiou, L. Borsten, M. J. Duff, L. J. Hughes, and S. Nagy, Yang-Mills Origin of Gravitational Symmetries, Phys. Rev. Lett. 113, 231606 (2014); D. Vaman and Y. P. Yao, Color kinematic symmetric (BCJ) numerators in a light-like gauge, J. High Energy Phys. 12 (2014) 036; A. Sabio Vera and M. A. Vázquez-Mozo, The double copy structure of soft gravitons, J. High Energy Phys. 03 (2015) 070.

[16] F. Cachazo, S. He, and E. Y. Yuan, Scattering of Massless Particles in Arbitrary Dimensions, Phys. Rev. Lett. 113, 171601 (2014); T. Adamo, E. Casali, and D. Skinner, Ambitwistor strings and the scattering equations at one loop, J. High Energy Phys. 04 (2014) 104; Y. Geyer, L. Mason, R. Monteiro, and P. Tourkine, Loop Integrands for Scattering Amplitudes from the Riemann Sphere, Phys.
Rev. Lett. 115, 121603 (2015); T. Adamo and E. Casali, Scattering equations, supergravity integrands, and pure spinors, J. High Energy Phys. 05 (2015) 120; S. He and E. Y. Yuan, One-loop scattering equations and amplitudes from forward limit, Phys. Rev. D 92, 105004 (2015); C. Baadsgaard, N. E. J. Bjerrum-Bohr, J. L. Bourjaily, S. Caron-Huot, P. H. Damgaard, and B. Feng, New representations of the perturbative S-matrix, arXiv:1509.02169.

[17] Z. Bern, J. J. M. Carrasco, H. Johansson, and R. Roiban, Five-Loop Four-Point Amplitude of $N=4$ Super-YangMills Theory, Phys. Rev. Lett. 109, 241602 (2012).

[18] Z. Bern, S. Davies, T. Dennen, Y.-t. Huang, and J. Nohle, Color-kinematics duality for pure Yang-Mills and gravity at one and two loops, Phys. Rev. D 92, 045041 (2015).

[19] Z. Bern, L. J. Dixon, and D. A. Kosower, A two loop four gluon helicity amplitude in QCD, J. High Energy Phys. 01 (2000) 027.

[20] Z. Bern, S. Davies, and T. Dennen, Enhanced ultraviolet cancellations in $\mathcal{N}=5$ supergravity at four loops, Phys. Rev. D 90, 105011 (2014).

[21] E. Cremmer, J. Scherk, and S. Ferrara, SU(4) invariant supergravity theory, Phys. Lett. 74B, 61 (1978).

[22] Z. Bern, S. Davies, T. Dennen, and Y.-t. Huang, Absence of Three-Loop Four-Point Divergences in $N=4$ Supergravity, Phys. Rev. Lett. 108, 201301 (2012).

[23] G. Bossard, P. S. Howe, K. S. Stelle, and P. Vanhove, The vanishing volume of $D=4$ superspace, Classical Quantum Gravity 28, 215005 (2011); G. Bossard, P. S. Howe, and K. S. Stelle, Anomalies and divergences in $N=4$ supergravity, Phys. Lett. B 719, 424 (2013); Invariants and divergences in half-maximal supergravity theories, J. High Energy Phys. 07 (2013) 117; Z. Bern, S. Davies, and T. Dennen, Ultraviolet structure of half-maximal supergravity with matter multiplets at two and three loops, Phys. Rev. D 88, 065007 (2013); The ultraviolet critical dimension of half-maximal supergravity at three loops, arXiv:1412.2441.

[24] Z. Bern, S. Davies, T. Dennen, A. V. Smirnov, and V. A. Smirnov, Ultraviolet Properties of $N=4$ Supergravity at Four Loops, Phys. Rev. Lett. 111, 231302 (2013).

[25] N. Marcus, Composite anomalies in supergravity, Phys. Lett. 157B, 383 (1985); J. J. M. Carrasco, R. Kallosh, R. Roiban, and A. A. Tseytlin, On the U(1) duality anomaly and the S-matrix of $N=4$ supergravity, J. High Energy Phys. 07 (2013) 029.

[26] Z. Bern, C. Cheung, H. H. Chi, S. Davies, L. Dixon, and J. Nohle, Evanescent Effects Can Alter Ultraviolet Divergences in Quantum Gravity without Physical Consequences, Phys. Rev. Lett. 115, 211301 (2015).

[27] S. H. Henry Tye and Y. Zhang, Dual identities inside the gluon and the graviton scattering amplitudes, J. High Energy Phys. 06 (2010) 071; 04 (2011) 114(E).

[28] Z. Bern, T. Dennen, Y.-t. Huang, and M. Kiermaier, Gravity as the square of gauge theory, Phys. Rev. D 82, 065003 (2010).

[29] N. E. J. Bjerrum-Bohr, P. H. Damgaard, T. Sondergaard, and P. Vanhove, The momentum kernel of gauge and gravity theories, J. High Energy Phys. 01 (2011) 001.

[30] J. J. M. Carrasco and H. Johansson, Generic multiloop methods and application to $N=4$ super-Yang-Mills, J. Phys. A 44, 454004 (2011); J. J. M. Carrasco, in Proceedings of the 
2014 Theoretical Advanced Study Institute in Elementary Particle Physics (World Scientific, Singapore, 2014).

[31] J. J. M. Carrasco, M. Chiodaroli, M. Günaydin, and R. Roiban, One-loop four-point amplitudes in pure and mattercoupled $\mathcal{N} \leq 4$ supergravity, J. High Energy Phys. 03 (2013) 056; N. E. J. Bjerrum-Bohr, T. Dennen, R. Monteiro, and D. O'Connell, Integrand oxidation and one-loop colourdual numerators in $N=4$ gauge theory, J. High Energy Phys. 07 (2013) 092; R. H. Boels, R. S. Isermann, R. Monteiro, and D. O'Connell, Colour-kinematics duality for one-loop rational amplitudes, J. High Energy Phys. 04 (2013) 107; S. He, R. Monteiro, and O. Schlotterer, String-inspired BCJ numerators for one-loop MHV amplitudes, J. High Energy Phys. 01 (2016) 171.

[32] C. R. Mafra and O. Schlotterer, Two-loop five-point amplitudes of super Yang-Mills and supergravity in pure spinor superspace, J. High Energy Phys. 10 (2015) $124 .$.

[33] M. L. Mangano and S. J. Parke, Multiparton amplitudes in gauge theories, Phys. Rep. 200, 301 (1991).

[34] G. 't Hooft and M. J. G. Veltman, Regularization and renormalization of gauge fields, Nucl. Phys. B44, 189 (1972); J. C. Collins, Renormalization (Cambridge University Press, Cambridge, 1984).

[35] Z. Bern and D. A. Kosower, The computation of loop amplitudes in gauge theories, Nucl. Phys. B379, 451 (1992).

[36] Z. Bern, L. J. Dixon, and D. A. Kosower, Two-loop $g \rightarrow g g$ splitting amplitudes in QCD, J. High Energy Phys. 08 (2004) 012.

[37] See Supplemental Material at http://link.aps.org/ supplemental/10.1103/PhysRevD.93.105015 for the arXiv version of this manuscript.

[38] M. H. Goroff and A. Sagnotti, Quantum gravity at two loops, Phys. Lett. 160B, 81 (1985); The ultraviolet behavior of Einstein gravity, Nucl. Phys. B266, 709 (1986); A. E. M. van de Ven, Two loop quantum gravity, Nucl. Phys. B378, 309 (1992).

[39] Z. Bern, C. Cheung, H.-H. Chi, S. Davies, L. Dixon, and J. Nohle (to be published).

[40] S. Weinberg, Infrared photons and gravitons, Phys. Rev. 140, B516 (1965); S. G. Naculich and H. J. Schnitzer, Eikonal methods applied to gravitational scattering amplitudes, J. High Energy Phys. 05 (2011) 087; S. G. Naculich, H. Nastase, and H. J. Schnitzer, All-loop infrared-divergent behavior of most-subleading-color gauge-theory amplitudes, J. High Energy Phys. 04 (2013) 114; R. Akhoury, R. Saotome, and G. Sterman, Collinear and soft divergences in perturbative quantum gravity, Phys. Rev. D 84, 104040 (2011).

[41] G. 't Hooft and M. J. G. Veltman, One loop divergencies in the theory of gravitation, Ann. Inst. Henri Poincaré A 20, 69 (1974); E. Sezgin and P. van Nieuwenhuizen, Renormalizability properties of antisymmetric tensor fields coupled to gravity, Phys. Rev. D 22, 301 (1980); E. S. Fradkin and A. A. Tseytlin, One loop infinities in dimensionally reduced supergravities, Phys. Lett. 137B, 357 (1984).

[42] D. M. Capper and M. J. Duff, Trace anomalies in dimensional regularization, Nuovo Cimento A 23, 173 (1974); Conformal anomalies and the renormalizability problem in quantum gravity, Phys. Lett. 53A, 361 (1975); H. S. Tsao, Conformal anomalies in a general background metric, Phys. Lett. 68B, 79 (1977); G. W. Gibbons, S. W. Hawking, and M. J. Perry, Path integrals and the indefiniteness of the gravitational action, Nucl. Phys. B138, 141 (1978); R. Critchley, Trace anomaly for gravitons, Phys. Rev. D 18, 1849 (1978); M. J. Duff and P. van Nieuwenhuizen, Quantum inequivalence of different field representations, Phys. Lett. 94B, 179 (1980); M. J. Duff, Twenty years of the Weyl anomaly, Classical Quantum Gravity 11, 1387 (1994). 